\title{
De-Dollarization and European Energy Policy: Testing Brent and WTI
}

\author{
Berk Cem \\ Department of Accounting and Information Systems, Istanbul Arel University, Istanbul, Turkey \\ Email: cemberk@arel.edu.tr
}

Received 1 July 2015; accepted 15 August 2015; published 18 August 2015

Copyright (C) 2015 by author and Scientific Research Publishing Inc. This work is licensed under the Creative Commons Attribution International License (CC BY). http://creativecommons.org/licenses/by/4.0/ c) (7) Open Access

\section{Abstract}

Historically most of the oil trade has been made with US Dollars. This has important practical implications. For example, US Dollar is always a reserve currency since it has to be obtained to trade oil. There were previous attempts to trade in Euros such as Iraq however recently there is more support from resource countries such as Russia, China and Iran. A de-dollarization campaign against petrodollar has already been started. This can be done by exporting oil, gas and gold with currencies other than US Dollar, such as Ruble, Yuan or Rial or most importantly an international reserve currency which could be Euros as a strong alternative to US Dollar. This is possible due to monetary policy practice of European Central Bank and support from European Union. This increases the value of Euro, decreases interest rates and helps European economy with increased interest on European assets. This paper investigates Brent and WTI for the changes in the value of major currencies. Brent is traditionally a European oil index, and oil is produced in North Sea whereas, WTI (West Texas Intermediate) is a Texas based US oil index. Both indices are used as international benchmarks of oil. The data for this research is daily between February 2011 and September 2014. The WTI and Brent are represented by variables from NYSE exchange traded funds namely Teucrium WTI Crude Oil ETF (WTI), and United States Brent Oil ETF(BRENT). The currencies analyzed for the study are EUR/USD, USD/CHF, USD/JPY, USD/RUB, USD/SAR and USD/ ZAR. The analysis includes unit root tests, vector autoregression (VAR), vector error correction model (VECM), cointegration and Granger Causality. Finally European Energy Policy implications, and opportunities and challenges of oil trade in Euros are discussed.

\section{Keywords}

De-Dollarization, Oil Exports, Euro Trading, Energy Policy, European Monetary Policy

\section{Introduction}

US Dollar is a very reliable currency; therefore most of world wealth is invested in Dollar based assets. There is 
a well known phenomenon in economics in explaining strong trust in US Dollar which is called "Dollarization”. Dollarization is either officially or unofficially increasing the use of Dollar in a country outside USA. There are many reasons for Dollarization, for example a country with unstable economic indicators will face currency attacks where the local currency loses value against the US Dollar. In addition in the times of inflation, the wealth held in local currency will lose its value against the fluctuating local prices so that the public would like to hold their assets in Dollar. This is mostly seen in flexible exchange rate regimes which are applied in most of the developed economies. The extreme case of this phenomenon is the use of US Dollar as the official currency of the country, thus abandoning the local currency. This paper investigates the effects of this phenomenon, especially the unofficial one in the field of international energy trade.

The balance of international trade is mostly determined with the exchange rates. Therefore it is important to have competitive power with local currency. The Dollar's most important competitor is Euro monetary union which is in question recently. The most important reason for this is the countries' loss of independent monetary policy actions where it is crucial especially in the time of economic recession. However, the countries have benefit when their currency has more usage. For dollarization, if most people hold Dollar, they will also hold assets issued by US to have a positive return from their investment. This is why most central banks, companies and individuals in the World buy US Treasury Bonds and other assets based on US Dollar which decreases the cost of funding for USA.

De-dollarization is the opposite process where less international trade is with currencies other than US Dollar. Euro is the most important competitor for Dollar, while there are other attempts to make the trade with Ruble or Yuan. Politically US would stand for trade in Dollar however economically there can be benefits to Europe and its trade partners if de-dollarization takes place.

This paper covers the international oil trade. However most of the international trade such as steel and other commodities takes place also in Dollar. In energy trade we see natural gas traded mostly in Dollar, and it is a similar case for oil. Oil is categorized due to its quality and where it is located with indices. The international indices are WTI, Brent, OPEC, Dubai, Oman, Urals and as well as more than 150 other indices however most reliable indices are WTI and Brent. WTI is West Texas Intermediate oil and it is Texas based oil, which has slightly more quality than Brent. Brent is a North Sea based oil. Both of these indices are globally used as international benchmarks of oil prices. In the study both of these two benchmarks are tested for de-dollarization.

The remainder of this paper is organized as follows. In Section 2, some of the recent and important works in this research area are presented. Then the methodology and research model are given in Section 3. The information on data, as well as research results is available in Section 4. In Section 5, some of the important findings of the study are discussed. In Section 6, policy and financial implications are discussed.

\section{Literature Review}

Tas and Togay investigate monetary policy for oil producing countries with a focus on Iraq. They analyzed the impact of exchange rate on oil revenue, the credibility of the central bank, oil dependence of the economy and asymmetric information between public and central bank. They find that due to asymmetric information, central bank policies have a major influence on public expectations. The use of dollarization also increases the credibility of the central bank. The economic growth expectation is higher and inflation expectation is lower when dollarization is applied. For the econometric model vector autoregression is developed for economic output and inflation and autoregression is used for oil prices (Tas \& Togay, 2010) [1].

Mete studies the relationship between liability dollarization and the exchange market pressure. The methodology used in this study is autoregressive distributed lag and Granger causality. The data is for the period from 1991:12 to 2006:08. The findings suggest a long-term relationship between exchange market and liability dollarization. Liability dollarization Granger causes EMP both in the short- and long-run, with no evidence of opposite causality. The weight of foreign currency liabilities in the commercial banks' balance sheets in Turkey induces a selling pressure in the exchange market as well as a fear of floating (Mete, 2012) [2].

Berrios studies the important case of Ecuador as it has official dollarization experience in 2000. This is done to battle against inflation and improves financial stability. The problem of economic stability was solved temporarily due to switching to dollarization regime however the lack of social and structural reforms remained as problems of this economy. Oil prices helped improve the economy as did the Ecuadorean workers abroad with remittances however the monetary policy is now affected less by Central Bank of Ecuador but by Federal Re- 
serve. This is also politically important as Venezuela's Chavez and Bolivia's Morales are shifting their oil and gas markets away from the United States to China and other customers (Berrios, 2006) [3].

Togay studies Kazakhstan as an oil producing country. It suffered seriously from the transition to market economy from 1991, when it declared its independence, to 1998. As of 2000, with the increase in oil prices, Kazakhstan's real gross domestic product increased significantly. However, the country also witnessed the emergence of an economic structure that is highly dependent on the oil sector for production, employment and foreign trade. How Kazakh economy's dependence on the petroleum sector can be reduced is a critical question concerning the development of both short term and long term economic policies. The study argues that the dependence of Kazakh economy on the oil sector is caused mainly by the problems concerning the use of foreign financial resources and the insufficiency of reform efforts. The study also discusses what role, if any, monetary policies could play in the short run in reducing Kazakhstan's dependence on oil. Finally, the study stresses the need for the Central Bank of Kazakhstan to move towards a system that could prevent Tenge's real value from destabilizing as a result of the changes in oil prices. The author suggests an alternative to dollarization as pegging the currency to a basket which includes oil (Togay, 2009) [4].

\section{Research Model}

The year of 1980 is treated as the beginning of the VAR concept with Sims's assumptions about the variable explained by its own delay and the delay of other variables were the starting point. In the VAR models the independent variables are delays of all the variables of this model. Assuming therefore that in the model there is a certain number of variables and taking into account the delays, the formula can be written as follows;

$$
y_{t}=A_{0} D_{t}+\sum A_{i} y_{i-1}+e_{t}, \quad t=1,2,3, \cdots, T
$$

where:

$y_{t}$-vector of observations on the current values of all $\mathrm{n}$ variables of the model,

$D_{t}$-vector of deterministic components of equations,

$A_{0}$-matrix of parameters for the vector variables $D_{t}$,

$A_{i}$ - parameters matrices at the delayed vactor variables $y_{t}$, matrices $A_{0}$ and $A_{i}$ do not contain zero elements,

$e_{t}$-vetor of stationary random noise,

$t$-observation number.

An important element while modeling by means of vector autoregression is a matter of determining the order of delays $p$. This choice can be made using the three criteria of information such as:

- Akaike (AIC): AIC $=-2 \ln (L) / T+2 k / T$,

- Schwart (BIC): $\mathrm{BIC}=-2 \ln (L) / T+k \ln (T) / T$,

- and Hannan-Qiunn (HQC): $\mathrm{HQC}=-2 \ln (L) / T+k \ln (\ln (T)) / T$,

where $L$ is the value of the likelihood function, and $k=n(d+n p)$ specifies the number of estimated parameters of the VAR model ( $d$-number of deterministic variables) (Mentel, 2012) [5].

This study uses a vector error correction model—short term (VECM) to distinguish the long-run relationship between the variables and the short-run dynamics (Engle and Granger 1987). The multivariate cointegration model is defined as follows:

$$
\Delta X_{t}=\mu+\sum \Gamma \Delta X_{t-I}+\Pi X_{t-I}+\partial_{t}+e_{t}
$$

where $X_{t}$ is a column vector of $p$ variables; $\Pi$ is a vector of constant terms; $\Gamma$ represents coefficient matrices; $\Delta$ is a difference operator; $\partial_{t}$ is the time trend. The coefficient matrix contains information about the long-run relationships (Permani, 587) [6].

To test Granger causality of two time series, Hong (2001) proposed one-sided asymptotically normal tests based on the cross correlation function (CCF) of standardized residuals. Consider two stationary time series $\left\{y_{i, t}, t=1, \cdots, T\right\}, i=1,2$, where $T$ is the sample size.

$$
y_{i, t}=E\left(y_{i, t} \mid I_{i, t-1}\right)+\varepsilon_{i, t}=E\left(y_{i, t} \mid I_{i, t-1}\right)+\sqrt{h_{i, t}} u_{i, t}, i=1,2
$$

where $I_{i, t}$ is the information set of time series $\left\{y_{i, t}\right\}$ available at period $t$, and $E\left(y_{i, t} \mid I_{i, t-1}\right)$ is the conditional mean of 
$y_{i, t}$. The residuals $\left\{\varepsilon_{i, t}=\sqrt{h_{i, t}} u_{i, t}\right\}$ may be heteroskedastic, where $u_{i, t}$ is the standardized residuals and $h_{i, t}$ is the conditional variance of $\varepsilon_{i, t}$. Denote $r_{j}$ as the cross correlation of standardized residuals2 $\left\{u_{i, t}, t=1,2, \cdots, T\right\}, i=1$, 2 with lag $j, \quad j=0, \pm 1, \pm 2, \cdots, \pm(T-1)$ :

$$
r_{j}=\left\{\begin{array}{l}
\frac{\sum_{t=j+1}^{T} u_{1, t} u_{2, t-j} / T}{\sqrt{\sum_{t=1}^{T} u_{1, t}^{2} / T} \sqrt{\sum_{t=1}^{T} u_{2, t}^{2} / T}}, j \geq 0 \\
\frac{\sum_{t=1-j}^{T} u_{1,1-j} u_{2, t} / T}{\sqrt{\sum_{t=1}^{T} u_{1, t}^{2} / T} \sqrt{\sum_{t=1}^{T} u_{2, t}^{2} / T}}, j<0
\end{array}\right.
$$

Then the unidirectional Granger causality test from $y_{1}$ to $y_{2}$ is defined as follows: equation

$$
H_{1}=\frac{T \sum_{j=1}^{T-1} k^{2}\left(\frac{j}{M}\right) r_{j}^{2}-C_{1 T}(k)}{\sqrt{2 D_{1 T}(k)}}
$$

The bidirectional Granger causality test is defined as follows: equation

$$
H_{2}=\frac{T \sum_{j=2-T}^{T-2} k^{2}\left(\frac{j}{M}\right) r_{j}^{2}-C_{2 T}(k)}{\sqrt{2 D_{2 T}(k)}}
$$

where $M$ is a positive integer and $k(x)$ is the kernel function. $C_{1 T}(k), C_{2 T}(k), D_{1 T}(k)$, and $D_{2 T}(k)$ are determined by $k(x)$ and the sample size $T$. Under certain regularity conditions, if $\left\{y_{i, t}\right\}, i=1,2$ are mutually independent, $H_{1}$ and $H_{2}$ are asymptotically distributed as the standard normal distribution $N(0,1) . H_{1}$ and $H_{2}$ are one-sided tests, so the upper-tailed $N(0,1)$ critical values should be used. The proposed tests allow for a flexible weight on CCF at each lag by some kernels, and Monte Carlo simulations show that the tests perform well (Lu et al. 2014) [7].

The two-step procedure provided by Engle and Granger (1987) is used to check the cointegration between oil price and exchange rates. And the cointegration equation is established as below.

$$
\begin{aligned}
& P O=w_{1}+\partial_{1} \times P E+\emptyset_{1} \\
& P E=w_{2}+\partial_{2} \times P O+\emptyset_{2}
\end{aligned}
$$

where $P O$ and $P E$ denote the crude oil price and exchange rate, respectively, and both $\varphi_{1}$ and $\varphi_{2}$ are residuals. If $\mathrm{ADF}$ test for $\varphi_{1}$ or $\varphi_{2}$ indicates that they are stationary, then we may say there is cointegration relationship between the crude oil price and exchange rate (Zhang \& Wei, 2010) [8].

\section{Data Analysis}

The data used in this study is WTI and Brent pricesused as NYSE exchange traded funds namely Teucrium WTI Crude Oil ETF (WTI), and United States Brent Oil ETF(BRENT)., and several currencies used as an exchange rate against the US Dollar to test Dollarization. The currencies used in this paper are Euro, Swiss Franc, Japanese Yen, Russian Ruble, Saudi Riyal, and South African Rand. The variables are used daily for the period between $2 / 23 / 2011$ and $9 / 19 / 2014$. The number of data is 891 .

Firstly the variables are checked to see whether they are stationary. This is done according to the Augmented Dickey Fuller and Philips Perron methodology. Both of the methods reveal that the variables WTI, EUR/USD, USD/CHF, USD/JPY, and USD/RUB are not stationary in level but they are stationary with their first differences, I(1). Whereas the variables Brent, USD/SAR, and USD/ZAR are stationary in level, I(0). The details of this unit root analysis are given in Table 1.

WTI is tested first for the dollarization effect. For choosing a proper auto regressive model for the series, lag order selection criteria is applied. 1 lag is suggested by Schwarz Information criterion. It is also suggested to use 2 lags according to other criterion. For the principle of parsimony VAR(1) is used, the Table 2 includes the full results of the test. 
Table 1. Unit root tests for the series.

\begin{tabular}{|c|c|c|c|c|}
\hline & ADF test statistic & Critical value & PP test statistic & Critical value \\
\hline WTI & -2.7255 & -2.8645 & -2.6454 & -2.8645 \\
\hline BRENT & -3.127 & -2.8645 & -3.0268 & -2.8645 \\
\hline EUR/USD & -2.0129 & -2.8645 & -1.9915 & -3.4148 \\
\hline USD/CHF & -2.4723 & -2.8645 & -2.3347 & -2.8645 \\
\hline USD/JPY & -2.2887 & -3.4148 & -2.2741 & -3.4148 \\
\hline USD/RUB & -2.3795 & -3.4148 & 1.4364 & -1.9412 \\
\hline USD/SAR & -6.7867 & -2.8645 & -6.8289 & -2.8645 \\
\hline USD/ZAR & -3.8628 & -3.4148 & -3.7434 & -3.4148 \\
\hline $\mathrm{D}(\mathrm{WTI})$ & -32.2203 & -1.9412 & -32.1916 & -1.9412 \\
\hline D(EUR/USD) & -25.6341 & -1.9412 & -25.5756 & -1.9412 \\
\hline $\mathrm{D}$ (USD/CHF) & -23.7428 & -1.9412 & -23.607 & -1.9412 \\
\hline D(USD/JPY) & -24.3202 & -1.9412 & -24.168 & -1.9412 \\
\hline D(USD/RUB) & -23.7097 & -1.9412 & -23.5654 & -1.9412 \\
\hline
\end{tabular}

Table 2. Lag length selection.

\begin{tabular}{|c|c|c|c|c|c|c|}
\hline \multicolumn{7}{|c|}{ VAR Lag Order Selection Criteria } \\
\hline \multicolumn{7}{|c|}{ Endogenous variables: D(WTI) USD_ZAR USD_SAR D(USD_RUB) D(USD_JPY) D(USD_CHF) D(EUR_USD) } \\
\hline \multicolumn{7}{|c|}{ Exogenous variables: $\mathrm{C}$} \\
\hline \multicolumn{7}{|c|}{ Date: $12 / 21 / 14$ Time: $16: 05$} \\
\hline \multicolumn{7}{|c|}{ Sample: 2/23/2011 9/19/2014 } \\
\hline \multicolumn{7}{|c|}{ Included observations: 891} \\
\hline Lag & $\log L$ & LR & FPE & AIC & SC & HQ \\
\hline 0 & 7083.971 & NA & $2.98 \mathrm{e}-16$ & -15.88546 & -15.84781 & -15.87107 \\
\hline 1 & 10712.02 & 7190.948 & $9.65 \mathrm{e}-20$ & -23.91924 & $-23.61804^{*}$ & -23.80412 \\
\hline 2 & 10808.77 & 190.2444 & $8.67 \mathrm{e}-20^{*}$ & $-24.02642^{*}$ & -23.46167 & $-23.81058^{*}$ \\
\hline 3 & 10838.12 & 57.24082 & $9.06 \mathrm{e}-20$ & -23.98230 & -23.15400 & -23.66573 \\
\hline 4 & 10871.71 & 65.00023 & $9.38 \mathrm{e}-20$ & -23.94772 & -22.85586 & -23.53042 \\
\hline 5 & 10899.45 & 53.23509 & $9.84 \mathrm{e}-20$ & -23.90000 & -22.54459 & -23.38197 \\
\hline 6 & 10938.02 & 73.41339 & $1.01 \mathrm{e}-19$ & -23.87658 & -22.25762 & -23.25783 \\
\hline 7 & 10975.14 & $70.07602^{*}$ & $1.04 \mathrm{e}-19$ & -23.84992 & -21.96740 & -23.13044 \\
\hline 8 & 11002.08 & 50.44250 & $1.09 \mathrm{e}^{-19}$ & -23.80041 & -21.65434 & -22.98020 \\
\hline
\end{tabular}

*indicates lag order selected by the criterion. LR: sequential modified LR test statistic (each test at 5\% level). FPE: Final prediction error. AIC: Akaike information criterion. SC: Schwarz information criterion. HQ: Hannan-Quinn information criterion.

The data is modelled with Vector Auto Regression (VAR). The results with substituted coefficients are given below for VAR(1).

VAR Model—Substituted Coefficients: 


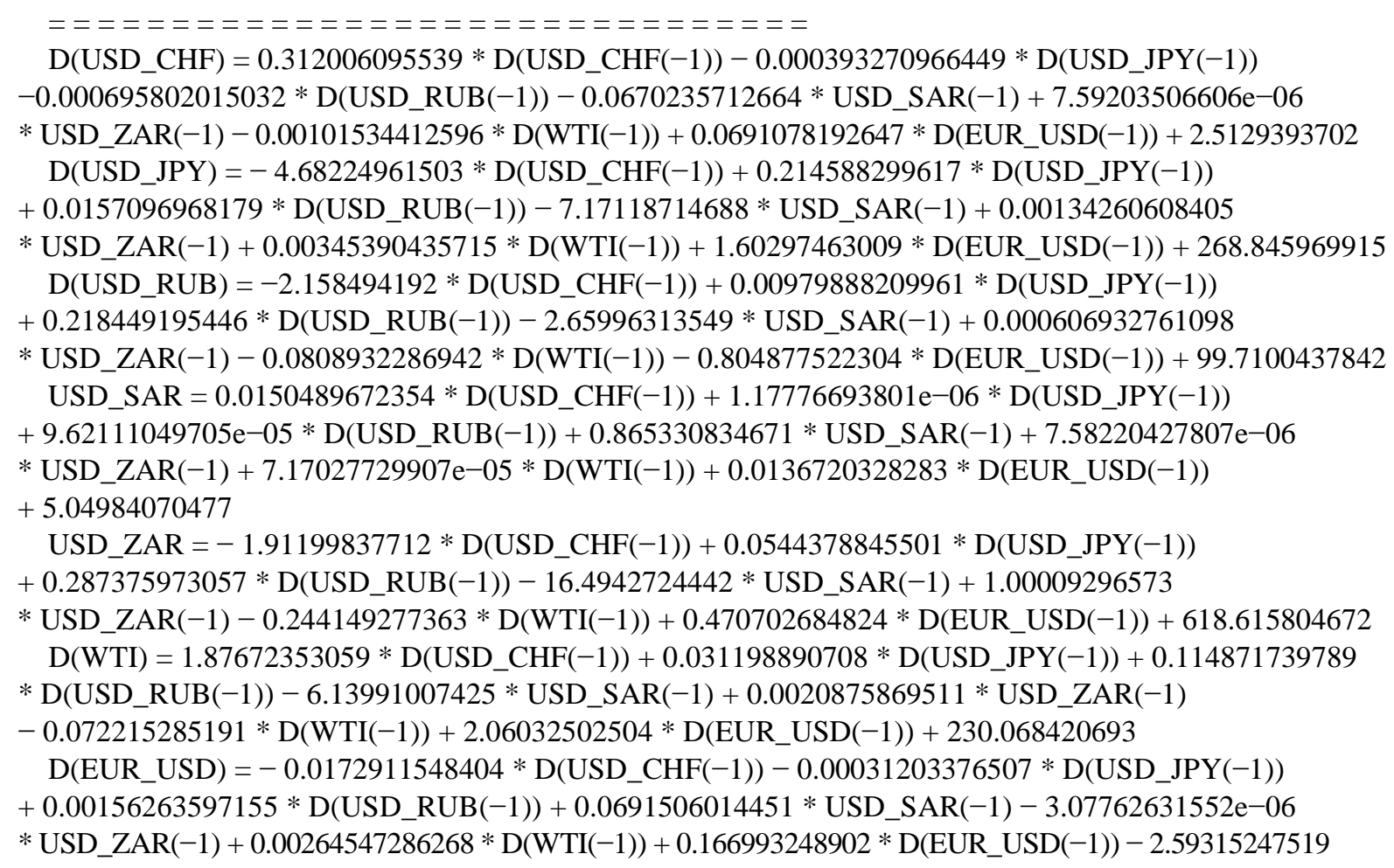

The accuracy of the model is tested by investigating the roots. According to the results there are no roots outside the unit circle, which indicates that the model is reliable. The results are given in Table 3.

Long-run effects for the model are tested according to Johansen Cointegration methodology. Accordingly, 6 cointegration equations are detected which is an indicator for long-run relationship. This is confirmed with both Trace and eigenvalue results which is given in Table 4.

The short run relationship between the variables is also analysed. For this a VECM (vector error correction model) is developed. The results with substituted coefficients are given below.

Table 3. Model stability.

Roots of characteristic polynomial

Endogenous variables: D(USD_CHF) D(USD_JPY) D(USD_RUB) USD_SAR USD_ZAR D(WTI) D(EUR_USD)

Exogenous variables: $\mathrm{C}$

Lag specification: 11

Date: 11/09/14 Time: $12: 40$

\begin{tabular}{cc} 
Root & Modulus \\
\hline 0.998883 & 0.998883 \\
0.865096 & 0.865096 \\
0.329510 & 0.329510 \\
$0.209716-0.045577 \mathrm{i}$ & 0.214611 \\
$0.209716+0.045577 \mathrm{i}$ & 0.214611 \\
0.140678 & 0.140678 \\
\hline
\end{tabular}

No root lies outside the unit circle. VAR satisfies the stability condition. 
Table 4. Cointegration results.

\begin{tabular}{|c|c|c|c|c|}
\hline \multicolumn{5}{|c|}{ Date: $11 / 09 / 14$ Time: $12: 40$} \\
\hline \multicolumn{5}{|c|}{ Sample (adjusted): 2/28/2011 9/19/2014 } \\
\hline \multicolumn{5}{|c|}{ Included observations: 897 after adjustments } \\
\hline \multicolumn{5}{|c|}{ Trend assumption: Linear deterministic trend } \\
\hline \multicolumn{5}{|c|}{ Series: D(USD_CHF) D(USD_JPY) D(USD_RUB) USD_SAR USD_ZAR D(WTI) D(EUR_USD) } \\
\hline \multicolumn{5}{|c|}{ Lags interval (in first differences): 1 to 1} \\
\hline \multicolumn{5}{|c|}{ Unrestricted Cointegration Rank Test (Trace) } \\
\hline Hypothesized & & Trace & 0.05 & \\
\hline No. of CE(s) & Eigenvalue & Statistic & Critical value & Prob. $^{* *}$ \\
\hline None $^{*}$ & 0.443660 & 1968.632 & 125.6154 & 1.0000 \\
\hline At most 1 & 0.336424 & 1442.653 & 95.75366 & 1.0000 \\
\hline At most $2^{*}$ & 0.322849 & 1074.783 & 69.81889 & 0.0001 \\
\hline At most $3^{*}$ & 0.318797 & 725.0778 & 47.85613 & 0.0001 \\
\hline At most $4^{*}$ & 0.306664 & 380.7238 & 29.79707 & 0.0001 \\
\hline At most $5^{*}$ & 0.055902 & 52.20582 & 15.49471 & 0.0000 \\
\hline At most 6 & 0.000675 & 0.605361 & 3.841466 & 0.4365 \\
\hline \multicolumn{5}{|c|}{ Unrestricted cointegration rank test (maximum eigenvalue) } \\
\hline Hypothesized & & Max-Eigen & 0.05 & \\
\hline No. of CE(s) & Eigenvalue & Statistic & Critical value & Prob. $^{* *}$ \\
\hline None* & 0.443660 & 525.9782 & 46.23142 & 0.0001 \\
\hline At most $1^{*}$ & 0.336424 & 367.8700 & 40.07757 & 0.0001 \\
\hline At most $2^{*}$ & 0.322849 & 349.7056 & 33.87687 & 0.0001 \\
\hline At most $3^{*}$ & 0.318797 & 344.3539 & 27.58434 & 0.0001 \\
\hline At most $4^{*}$ & 0.306664 & 328.5180 & 21.13162 & 0.0001 \\
\hline At most $5^{*}$ & 0.055902 & 51.60046 & 14.26460 & 0.0000 \\
\hline At most 6 & 0.000675 & 0.605361 & 3.841466 & 0.4365 \\
\hline
\end{tabular}

Trace test indicates 6 cointegrating eqn(s) at the 0.05 level. ${ }^{*}$ denotes rejection of the hypothesis at the 0.05 level. ${ }^{* *}$ MacKinnon-Haug-Michelis (1999) p-values. Max-eigenvalue test indicates 6 cointegrating eqn(s) at the 0.05 level.

$\mathrm{D}(\mathrm{WTI}, 2)=-0.519261875184 *\left(\mathrm{D}(\mathrm{WTI}(-1))-95.9358640088 * \mathrm{D}\left(\mathrm{USD} \_\mathrm{CHF}(-1)\right)+0.119792107105\right.$

* D(USD_JPY(-1)) + 0.9255301098 * D(USD_RUB $(-1))-1.85232125886 *$ USD_SAR $(-1)$

$-0.00148561163795 *$ USD_ZAR(-1) $-88.7053247156 *$ D(EUR_USD $(-1))+69.5889716493)$

$-0.272814645055 * \mathrm{D}(\mathrm{WTI}(-1), 2)-26.193050652 * \mathrm{D}\left(\mathrm{USD} \_\mathrm{CHF}(-1), 2\right)+0.0762511319927$

* D(USD_JPY(-1),2) + 0.226696841672 * D(USD_RUB $(-1), 2)-64.3932289086$ * D(USD_SAR(-1))

$+0.132383345795 * \mathrm{D}\left(\mathrm{USD} \_Z A R(-1)\right)-23.7704697493 * \mathrm{D}\left(\mathrm{EUR} \_\right.$USD $\left.(-1), 2\right)-0.00688107606467$

$\mathrm{D}(\mathrm{USD}$ _CHF,2 $)=0.000171119116358 *(\mathrm{D}(\mathrm{WTI}(-1))-95.9358640088 * \mathrm{D}(\mathrm{USD}$ _CHF $(-1))$

$+0.119792107105 * \mathrm{D}\left(\mathrm{USD} \_J P Y(-1)\right)+0.9255301098 * \mathrm{D}\left(\mathrm{USD} \_\mathrm{RUB}(-1)\right)-1.85232125886$ 
* USD_SAR $(-1)-0.00148561163795$ * USD_ZAR $(-1)-88.7053247156$ * D(EUR_USD $(-1))$

$+69.5889716493)-0.000736442856295 * \mathrm{D}(\mathrm{WTI}(-1), 2)-0.250866893109 * \mathrm{D}\left(\mathrm{USD} \_\mathrm{CHF}(-1), 2\right)$

$-0.000259578309291 * \mathrm{D}\left(\mathrm{USD} \_J P Y(-1), 2\right)+0.000512561377737 * \mathrm{D}\left(\mathrm{USD} \_\mathrm{RUB}(-1), 2\right)$

$-0.274640512389 *$ D(USD_SAR(-1) $)-0.00143999890734 *$ D(USD_ZAR $(-1))+0.0520197724068$

* D(EUR_USD $(-1), 2)+7.68809283598 \mathrm{e}-05$

D(USD_JPY,2) $=0.0581276197261 *\left(\mathrm{D}(\mathrm{WTI}(-1))-95.9358640088 * \mathrm{D}\left(\mathrm{USD} \_\mathrm{CHF}(-1)\right)\right.$

$+0.119792107105 *$ D(USD_JPY $(-1))+0.9255301098 *$ D(USD_RUB $(-1))-1.85232125886 *$ USD_SAR( $(-1)$

$-0.00148561163795 *$ USD_ZAR(-1) $-88.7053247156 *$ D(EUR_USD $(-1))+69.5889716493)$

$-0.0442979458622 * \mathrm{D}(\mathrm{WTI}(-1), 2)+0.198239647612 * \mathrm{D}\left(\mathrm{USD} \_\mathrm{CHF}(-1), 2\right)-0.348014699855$

* D(USD_JPY $(-1), 2)+0.0230388663407 *$ D(USD_RUB $(-1), 2)-1.08896576986 *$ D(USD_SAR( $(-1))$

$-0.0624046693192 * \mathrm{D}\left(\mathrm{USD} \_Z A R(-1)\right)+2.93687317617 * \mathrm{D}\left(\mathrm{EUR} \_\mathrm{USD}(-1), 2\right)+0.00510984723234$

$\mathrm{D}\left(\mathrm{USD} \_\mathrm{RUB}, 2\right)=-0.156854702674 *\left(\mathrm{D}(\mathrm{WTI}(-1))-95.9358640088 * \mathrm{D}\left(\mathrm{USD} \_\mathrm{CHF}(-1)\right)\right.$

$+0.119792107105 * \mathrm{D}\left(\mathrm{USD} \_J P Y(-1)\right)+0.9255301098 * \mathrm{D}\left(\mathrm{USD} \_\mathrm{RUB}(-1)\right)-1.85232125886$

* USD_SAR(-1) - 0.00148561163795 * USD_ZAR(-1) - 88.7053247156 * D(EUR_USD $(-1))$

$+69.5889716493)+0.0605875934627 * \mathrm{D}(\mathrm{WTI}(-1), 2)-12.0786308153 * \mathrm{D}\left(\mathrm{USD} \_\mathrm{CHF}(-1), 2\right)$

$+0.0320227654077 * \mathrm{D}(\mathrm{USD}$ JPY $(-1), 2)-0.29716230983 * \mathrm{D}\left(\mathrm{USD} \_\mathrm{RUB}(-1), 2\right)-3.32631101652$

* D(USD_SAR(-1)) $-0.0504024011297 *$ D(USD_ZAR(-1)) $-9.08398088941 *$ D(EUR_USD $(-1), 2)$

$+0.00228941483039$

$\mathrm{D}\left(\mathrm{USD} \_S A R\right)=3.42196267043 \mathrm{e}-05 *\left(\mathrm{D}(\mathrm{WTI}(-1))-95.9358640088 * \mathrm{D}\left(\mathrm{USD} \_\mathrm{CHF}(-1)\right)\right.$

$+0.119792107105 * \mathrm{D}\left(\mathrm{USD} \_J P Y(-1)\right)+0.9255301098 * \mathrm{D}\left(\mathrm{USD} \_\mathrm{RUB}(-1)\right)-1.85232125886$

* USD_SAR(-1) - 0.00148561163795 * USD_ZAR(-1) - 88.7053247156 * D(EUR_USD $(-1))$

$+69.5889716493)+1.28025171114 \mathrm{e}-05 * \mathrm{D}(\mathrm{WTI}(-1), 2)+0.0204755773799 * \mathrm{D}(\mathrm{USD}$ _CHF(-1),2)

$-2.89427532102 \mathrm{e}-05 * \mathrm{D}\left(\mathrm{USD} \_J P Y(-1), 2\right)+2.89084334439 \mathrm{e}-05 * \mathrm{D}\left(\mathrm{USD} \_\mathrm{RUB}(-1), 2\right)-0.191937399923$

* D(USD_SAR(-1)) + 3.08594550923e-05 * D(USD_ZAR(-1) $+0.0166830927004 *$ D(EUR_USD $(-1), 2)$

$-5.96351578023 \mathrm{e}-06$

$\mathrm{D}\left(\mathrm{USD} \_Z A R\right)=-0.259836107277 *\left(\mathrm{D}(\mathrm{WTI}(-1))-95.9358640088 * \mathrm{D}\left(\mathrm{USD} \_\mathrm{CHF}(-1)\right)\right.$

$+0.119792107105 * \mathrm{D}\left(\mathrm{USD} \_J P Y(-1)\right)+0.9255301098 * \mathrm{D}\left(\mathrm{USD} \_\mathrm{RUB}(-1)\right)-1.85232125886$

* USD_SAR(-1) - 0.00148561163795 * USD_ZAR(-1) - 88.7053247156 * D(EUR_USD $(-1))$

$+69.5889716493)+0.0886317280982 * \mathrm{D}(\mathrm{WTI}(-1), 2)-19.1181962511 * \mathrm{D}\left(\mathrm{USD} \_\mathrm{CHF}(-1), 2\right)$

$+0.0757362969014 * \mathrm{D}\left(\mathrm{USD} \_J P Y(-1), 2\right)+0.053065107846 * \mathrm{D}\left(\mathrm{USD} \_\mathrm{RUB}(-1), 2\right)+7.8079217173$

* D(USD_SAR(-1)) +0.179483952792 * D(USD_ZAR(-1)) -11.7945916109 * D(EUR_USD(-1),2)

$+0.0363554650575$

$\mathrm{D}($ EUR_USD,2 $)=0.00550218549673 *\left(\mathrm{D}(\mathrm{WTI}(-1))-95.9358640088 * \mathrm{D}\left(\mathrm{USD} \_\mathrm{CHF}(-1)\right)\right.$

$+0.119792107105 * \mathrm{D}\left(\mathrm{USD} \_J P Y(-1)\right)+0.9255301098 * \mathrm{D}\left(\mathrm{USD} \_\mathrm{RUB}(-1)\right)-1.85232125886$

* USD_SAR(-1) - 0.00148561163795 * USD_ZAR(-1) - 88.7053247156 * D(EUR_USD $(-1))$

$+69.5889716493)-0.00191436098299 * \mathrm{D}(\mathrm{WTI}(-1), 2)+0.328953767962 * \mathrm{D}\left(\mathrm{USD} \_\mathrm{CHF}(-1), 2\right)$

$-0.000949782849437 * \mathrm{D}\left(\mathrm{USD} \_J P Y(-1), 2\right)-0.00169789326266 * \mathrm{D}\left(\mathrm{USD} \_\mathrm{RUB}(-1), 2\right)+0.30770839636$

* D(USD_SAR(-1)) $+0.00223471374513 *$ D(USD_ZAR(-1)) -0.0688820160086 * D(EUR_USD $(-1), 2)$

$-0.000107762148972$

Finally for analysing short run causality under $5 \%$ level of significance Granger test is applied. According to the results only USD/CHF, USD/SAR, USD/ZAR, and EUR/USD Granger causes WTI. Whereas USD/JPY, and USD/RUB does not Granger Cause WTI. WTI on the other hand Granger causes, USD/CHF, USD/RUB, USD/ZAR and EUR/USD.The results are given in Table 5.

Brent is also tested first for the dollarization effect. For choosing a proper auto regressive model for the series, lag order selection criteria is applied. 1 lag is suggested by Schwarz Information criterion. It is also suggested to use more lags according to other criterion. For the principle of parsimony VAR(1) is used, the Table 6 includes the full results of the test.

The data is modelled with Vector Auto Regression (VAR). The results with substituted coefficients are given below for VAR(1).

BRENT $=0.967754941515 *$ BRENT $(-1)+0.00422889071678 *$ USD_ZAR $(-1)-8.76919146378$

* USD_SAR(-1) $-0.0257253653909 *$ D(USD_RUB $(-1))+0.0410936111944 *$ D(USD_JPY $(-1))$

$-5.46819809971 *$ D(USD_CHF $(-1))-4.86926399521 *$ D(EUR_USD $(-1))+329.803268446$ 
Table 5. Granger causality results.

\begin{tabular}{|c|c|c|c|}
\hline \multicolumn{4}{|c|}{ VEC Granger Causality/Block Exogeneity Wald Tests } \\
\hline \multicolumn{4}{|c|}{ Date: 11/09/14 Time: $12: 52$} \\
\hline \multicolumn{4}{|c|}{ Sample: 2/23/2011 9/19/2014 } \\
\hline \multicolumn{4}{|c|}{ Included observations: 897} \\
\hline \multicolumn{4}{|c|}{ Dependent variable: D(WTI,2) } \\
\hline Excluded & Chi-sq & $\mathrm{df}$ & Prob. \\
\hline D(USD_CHF,2) & 19.74048 & 1 & 0.0000 \\
\hline D(USD_JPY,2) & 3.075048 & 1 & 0.0795 \\
\hline D(USD_RUB,2) & 3.104736 & 1 & 0.0781 \\
\hline D(USD_SAR) & 4.398922 & 1 & 0.0360 \\
\hline D(USD_ZAR) & 12.38486 & 1 & 0.0004 \\
\hline D(EUR_USD,2) & 24.16334 & 1 & 0.0000 \\
\hline All & 71.72270 & 6 & 0.0000 \\
\hline \multicolumn{4}{|c|}{ Dependent variable: D(USD_CHF,2) } \\
\hline Excluded & Chi-sq & df & Prob. \\
\hline $\mathrm{D}(\mathrm{WTI}, 2)$ & 5.771782 & 1 & 0.0163 \\
\hline D(USD_JPY,2) & 0.497868 & 1 & 0.4804 \\
\hline D(USD_RUB,2) & 0.221740 & 1 & 0.6377 \\
\hline D(USD_SAR) & 1.117926 & 1 & 0.2904 \\
\hline D(USD_ZAR) & 20.47231 & 1 & 0.0000 \\
\hline D(EUR_USD,2) & 1.616726 & 1 & 0.2035 \\
\hline All & 28.45028 & 6 & 0.0001 \\
\hline \multicolumn{4}{|c|}{ Dependent variable: D(USD_JPY,2) } \\
\hline Excluded & Chi-sq & df & Prob. \\
\hline $\mathrm{D}(\mathrm{WTI}, 2)$ & 2.444954 & 1 & 0.1179 \\
\hline D(USD_CHF,2) & 0.001850 & 1 & 0.9657 \\
\hline D(USD_RUB,2) & 0.052450 & 1 & 0.8189 \\
\hline D(USD_SAR) & 0.002058 & 1 & 0.9638 \\
\hline D(USD_ZAR) & 4.501409 & 1 & 0.0339 \\
\hline D(EUR_USD,2) & 0.603312 & 1 & 0.4373 \\
\hline All & 9.509538 & 6 & 0.1469 \\
\hline \multicolumn{4}{|c|}{ Dependent variable: D(USD_RUB,2) } \\
\hline Excluded & Chi-sq & $\mathrm{df}$ & Prob. \\
\hline $\mathrm{D}(\mathrm{WTI}, 2)$ & 37.31471 & 1 & 0.0000 \\
\hline D(USD_CHF,2) & 56.01701 & 1 & 0.0000 \\
\hline D(USD_JPY,2) & 7.237291 & 1 & 0.0071 \\
\hline
\end{tabular}




\section{Continued}

\begin{tabular}{|c|c|c|c|}
\hline D(USD_SAR) & 0.156636 & 1 & 0.6923 \\
\hline D(USD_ZAR) & 23.95664 & 1 & 0.0000 \\
\hline D(EUR_USD,2) & 47.09045 & 1 & 0.0000 \\
\hline All & 108.0287 & 6 & 0.0000 \\
\hline \multicolumn{4}{|c|}{ Dependent variable: D(USD_SAR) } \\
\hline Excluded & Chi-sq & $\mathrm{df}$ & Prob. \\
\hline $\mathrm{D}(\mathrm{WTI}, 2)$ & 0.111844 & 1 & 0.7381 \\
\hline D(USD_CHF,2) & 10.80600 & 1 & 0.0010 \\
\hline D(USD_JPY,2) & 0.396868 & 1 & 0.5287 \\
\hline D(USD_RUB,2) & 0.045226 & 1 & 0.8316 \\
\hline D(USD_ZAR) & 0.602848 & 1 & 0.4375 \\
\hline D(EUR_USD,2) & 10.66207 & 1 & 0.0011 \\
\hline All & 15.30294 & 6 & 0.0180 \\
\hline \multicolumn{4}{|c|}{ Dependent variable: D(USD_ZAR) } \\
\hline Excluded & Chi-sq & $\mathrm{df}$ & Prob. \\
\hline $\mathrm{D}(\mathrm{WTI}, 2)$ & 6.504528 & 1 & 0.0108 \\
\hline D(USD_CHF,2) & 11.43149 & 1 & 0.0007 \\
\hline D(USD_JPY,2) & 3.297548 & 1 & 0.0694 \\
\hline D(USD_RUB,2) & 0.184916 & 1 & 0.6672 \\
\hline D(USD_SAR) & 0.070301 & 1 & 0.7909 \\
\hline D(EUR_USD,2) & 6.466524 & 1 & 0.0110 \\
\hline All & 15.73929 & 6 & 0.0152 \\
\hline \multicolumn{4}{|c|}{ Dependent variable: D(EUR_USD,2) } \\
\hline Excluded & Chi-sq & $\mathrm{df}$ & Prob. \\
\hline $\mathrm{D}(\mathrm{WTI}, 2)$ & 28.47086 & 1 & 0.0000 \\
\hline D(USD_CHF,2) & 31.75371 & 1 & 0.0000 \\
\hline D(USD_JPY,2) & 4.865722 & 1 & 0.0274 \\
\hline D(USD_RUB,2) & 1.776208 & 1 & 0.1826 \\
\hline D(USD_SAR) & 1.024433 & 1 & 0.3115 \\
\hline D(USD_ZAR) & 35.99213 & 1 & 0.0000 \\
\hline All & 90.92641 & 6 & 0.0000 \\
\hline
\end{tabular}

USD_ZAR $=-0.0112074749165 *$ BRENT $(-1)+1.00090962538 *$ USD_ZAR(-1) -15.2295526003

* USD_SAR(-1) + $0.30979344778 *$ D(USD_RUB $(-1))+0.0723977861224 *$ D(USD_JPY $(-1))$

$-5.19441817566 *$ D(USD_CHF $(-1))-0.564041021918 *$ D(EUR_USD $(-1))+571.567612336$ 
USD_SAR $=1.54632648314 \mathrm{e}-05$ * BRENT $(-1)+5.94625534225 \mathrm{e}-06$ * USD_ZAR $(-1)+0.863316042306$

* USD_SAR $(-1)+0.000108045704063$ * D(USD_RUB $(-1))-6.0709975285 \mathrm{e}-06$ * D(USD_JPY $(-1))$

$+0.0163743337872 * \mathrm{D}\left(\mathrm{USD} \_\mathrm{CHF}(-1)\right)+0.0141041901343 * \mathrm{D}\left(\mathrm{EUR} \_\mathrm{USD}(-1)\right)+5.12492069594$

$\mathrm{D}($ USD_RUB $)=-0.00820891547703 *$ BRENT $(-1)+0.00139315529542 *$ USD_ZAR $(-1)$

$-1.63390757161 *$ USD_SAR $(-1)+0.219074075644 *$ D(USD_RUB $(-1))+0.0164786530759$

* D(USD_JPY(-1)) - 3.3795186475 * D(USD_CHF(-1)) - 1.19509139045 * D(EUR_USD $(-1))$

$+61.4927143212$

$\mathrm{D}\left(\mathrm{USD} \_J P Y\right)=0.000836815217278 *$ BRENT $(-1)+0.00125325610824 *$ USD_ZAR $(-1)$

$-7.28066235711 *$ USD_SAR( $(-1)+0.0164189053626 *$ D(USD_RUB $(-1))+0.214224213206$

* D(USD_JPY(-1)) $-4.61567699898 *$ D(USD_CHF(-1)) + 1.62476055711 * D(EUR_USD $(-1))$

$+272.925677109$

$\mathrm{D}\left(\mathrm{USD} \_\mathrm{CHF}\right)=-0.000158558662585 *$ BRENT $(-1)+2.38289193387 \mathrm{e}-05 *$ USD_ZAR $(-1)$

$-0.0466477963372 *$ USD_SAR $(-1)-0.000771975457384 *$ D(USD_RUB $(-1))-0.00030042303977$

* D(USD_JPY $(-1))+0.295031772201 *$ D(USD_CHF $(-1))+0.0636248725389 *$ D(EUR_USD $(-1))$

$+1.75376618889$

$\mathrm{D}($ EUR_USD $)=0.000217697119514 *$ BRENT $(-1)-2.29673753836 \mathrm{e}-05 *$ USD_ZAR $(-1)$

$+0.0424493241433 *$ USD_SAR(-1) + $0.00146538929377 *$ D(USD_RUB $(-1))-0.000522250745891$

* D(USD_JPY(-1)) $+0.0211332700296 *$ D(USD_CHF(-1)) $+0.179219583762 *$ D(EUR_USD $(-1))$

$-1.59883355459$

The accuracy of the model is tested by investigating the roots. According to the results there are no roots outside the unit circle, which indicates that the model is reliable. The results are given in Table 7.

Long-run effects for the model are tested according to Johansen Cointegration methodology. Accordingly, 5 cointegration equations are detected which is an indicator for long-run relationship. This is confirmed with both Trace and Eigenvalue results which is given in Table 8.

The short run relationship between the variables is also analysed. For this a VECM (vector error correction model) is developed. The results with substituted coefficients are given below.

Table 6. Lag length selection.

\begin{tabular}{|c|c|c|c|c|c|c|}
\hline \multicolumn{7}{|c|}{ VAR Lag Order Selection Criteria } \\
\hline \multicolumn{7}{|c|}{ Endogenous variables: BRENT USD_ZAR USD_SAR D(USD_RUB) D(USD_JPY) D(USD_CHF) D(EUR_USD) } \\
\hline \multicolumn{7}{|c|}{ Exogenous variables: $\mathrm{C}$} \\
\hline \multicolumn{7}{|c|}{ Date: 12/21/14 Time: $16: 16$} \\
\hline \multicolumn{7}{|c|}{ Sample: 2/23/2011 9/19/2014 } \\
\hline \multicolumn{7}{|c|}{ Included observations: 891} \\
\hline Lag & $\log L$ & LR & FPE & AIC & SC & HQ \\
\hline 0 & 5963.302 & NA & $3.68 \mathrm{e}-15$ & -13.36993 & -13.33228 & -13.35554 \\
\hline 1 & 10780.55 & 9548.000 & $8.28 \mathrm{e}-20$ & -24.07307 & $-23.77187^{*}$ & -23.95796 \\
\hline 2 & 10882.52 & 200.4925 & $7.35 \mathrm{e}-20$ & -24.19196 & -23.62720 & $-23.97611^{*}$ \\
\hline 3 & 10942.47 & 116.9507 & $7.17 \mathrm{e}-20^{*}$ & $-24.21655^{*}$ & -23.38824 & -23.89998 \\
\hline 4 & 10974.57 & 62.11279 & $7.45 \mathrm{e}-20$ & -24.17862 & -23.08676 & -23.76132 \\
\hline 5 & 11002.14 & 52.91097 & $7.82 \mathrm{e}-20$ & -24.13051 & -22.77510 & -23.61249 \\
\hline 6 & 11042.61 & 77.02092 & $7.97 \mathrm{e}-20$ & -24.11135 & -22.49239 & -23.49260 \\
\hline 7 & 11079.88 & $70.36572^{*}$ & $8.19 \mathrm{e}-20$ & -24.08503 & -22.20252 & -23.36555 \\
\hline 8 & 11107.12 & 50.98304 & $8.60 \mathrm{e}-20$ & -24.03617 & -21.89011 & -23.21597 \\
\hline
\end{tabular}

*indicates lag order selected by the criterion. LR: sequential modified LR test statistic (each test at $5 \%$ level). FPE: Final prediction error. AIC: Akaike information criterion. SC: Schwarz information criterion. HQ: Hannan-Quinn information criterion. 
Table 7. Model stability.

\begin{tabular}{cc}
\hline \multicolumn{3}{c}{ Roots of Characteristic Polynomial } \\
\hline Endogenous variables: BRENT USD_ZAR USD_SAR D(USD_RUB) D(USD_JPY) D(USD_CHF) D(EUR_USD) \\
\hline \\
Exogenous variables: C \\
\hline Lag specification: 11 \\
\hline 0.998877 & Date: $12 / 21 / 14$ Time: $16: 19$ \\
0.968562 & Modulus \\
0.864908 & 0.998877 \\
0.332298 & 0.968562 \\
0.213925 & 0.864908 \\
$0.180480-0.036901 \mathrm{i}$ & 0.332298 \\
$0.180480+0.036901 \mathrm{i}$ & 0.213925 \\
\hline
\end{tabular}

No root lies outside the unit circle. VAR satisfies the stability condition.

Table 8. Cointegration results.

\begin{tabular}{|c|c|c|c|c|}
\hline \multicolumn{5}{|c|}{ Date: 12/21/14 Time: $16: 22$} \\
\hline \multicolumn{5}{|c|}{ Sample (adjusted): 2/28/2011 9/19/2014 } \\
\hline \multicolumn{5}{|c|}{ Included observations: 897 after adjustments } \\
\hline \multicolumn{5}{|c|}{ Trend assumption: Linear deterministic trend } \\
\hline \multicolumn{5}{|c|}{ Series: BRENT USD_ZAR USD_SAR D(USD_RUB) D(USD_JPY) D(USD_CHF) D(EUR_USD) } \\
\hline \multicolumn{5}{|c|}{ Lags interval (in first differences): 1 to 1} \\
\hline \multicolumn{5}{|c|}{ Unrestricted Cointegration Rank Test (Trace) } \\
\hline Hypothesized & & Trace & 0.05 & \\
\hline No. of CE(s) & Eigenvalue & Statistic & Critical Value & Prob. $^{* *}$ \\
\hline None $^{*}$ & 0.369649 & 1502.813 & 125.6154 & 1.0000 \\
\hline At most $1^{*}$ & 0.320385 & 1088.867 & 95.75366 & 0.0001 \\
\hline At most $2^{*}$ & 0.320105 & 742.4192 & 69.81889 & 0.0001 \\
\hline At most $3^{*}$ & 0.307898 & 396.3412 & 47.85613 & 0.0001 \\
\hline At most $4^{*}$ & 0.057232 & 66.22534 & 29.79707 & 0.0000 \\
\hline At most 5 & 0.014063 & 13.36032 & 15.49471 & 0.1022 \\
\hline At most 6 & 0.000732 & 0.656455 & 3.841466 & 0.4178 \\
\hline \multicolumn{5}{|c|}{ Unrestricted Cointegration Rank Test (Maximum Eigenvalue) } \\
\hline Hypothesized & & Max-Eigen & 0.05 & \\
\hline No. of CE(s) & Eigenvalue & Statistic & Critical Value & Prob. $^{* *}$ \\
\hline None $^{*}$ & 0.369649 & 413.9461 & 46.23142 & 0.0001 \\
\hline At most $1^{*}$ & 0.320385 & 346.4474 & 40.07757 & 0.0001 \\
\hline At most $2^{*}$ & 0.320105 & 346.0780 & 33.87687 & 0.0001 \\
\hline At most $3^{*}$ & 0.307898 & 330.1158 & 27.58434 & 0.0001 \\
\hline At most $4^{*}$ & 0.057232 & 52.86502 & 21.13162 & 0.0000 \\
\hline At most 5 & 0.014063 & 12.70386 & 14.26460 & 0.0869 \\
\hline At most 6 & 0.000732 & 0.656455 & 3.841466 & 0.4178 \\
\hline
\end{tabular}

Trace test indicates 5 cointegrating eqn(s) at the 0.05 level. " denotes rejection of the hypothesis at the 0.05 level. ${ }^{* *}$ MacKinnon-Haug-Michelis (1999) p-values. Max-eigenvalue test indicates 5 cointegrating eqn(s) at the 0.05 level. 
$\mathrm{D}(\mathrm{BRENT})=\mathrm{A}(1,1) *(\mathrm{~B}(1,1) * \mathrm{BRENT}(-1)+\mathrm{B}(1,2) * \mathrm{USD} Z \mathrm{ZAR}(-1)+\mathrm{B}(1,3) *$ USD_SAR$(-1)$

$+\mathrm{B}(1,4) * \mathrm{D}(\mathrm{USD}$ RUB $(-1))+\mathrm{B}(1,5) * \mathrm{D}\left(\mathrm{USD} \_J P Y(-1)\right)+\mathrm{B}(1,6) * \mathrm{D}\left(\mathrm{USD} \_\mathrm{CHF}(-1)\right)+\mathrm{B}(1,7)$

* D(EUR_USD $(-1))+\mathrm{B}(1,8))+\mathrm{C}(1,1) * \mathrm{D}(\mathrm{BRENT}(-1))+\mathrm{C}(1,2) * \mathrm{D}(\mathrm{USD}$ ZZAR $(-1))+\mathrm{C}(1,3)$

* D(USD_SAR $(-1))+\mathrm{C}(1,4) * \mathrm{D}\left(\mathrm{USD} \_\mathrm{RUB}(-1), 2\right)+\mathrm{C}(1,5) * \mathrm{D}(\mathrm{USD}$ JPY $(-1), 2)+\mathrm{C}(1,6)$

* D(USD_CHF $(-1), 2)+\mathrm{C}(1,7) * \mathrm{D}\left(\mathrm{EUR} \_U S D(-1), 2\right)+\mathrm{C}(1,8)$

$\mathrm{D}\left(\mathrm{USD} \_\mathrm{ZAR}\right)=\mathrm{A}(2,1) *(\mathrm{~B}(1,1)$ * BRENT $(-1)+\mathrm{B}(1,2)$ * USD_ZAR $(-1)+\mathrm{B}(1,3)$ * USD_SAR $(-1)$

$+\mathrm{B}(1,4) * \mathrm{D}\left(\mathrm{USD} \_\mathrm{RUB}(-1)\right)+\mathrm{B}(1,5) * \mathrm{D}\left(\mathrm{USD} \_\mathrm{JPY}(-1)\right)+\mathrm{B}(1,6) * \mathrm{D}(\mathrm{USD}$ CHF $(-1))+\mathrm{B}(1,7)$

* D(EUR_USD $(-1))+\mathrm{B}(1,8))+\mathrm{C}(2,1) * \mathrm{D}(\mathrm{BRENT}(-1))+\mathrm{C}(2,2) * \mathrm{D}\left(\mathrm{USD} \_Z A R(-1)\right)+\mathrm{C}(2,3)$

* D(USD_SAR $(-1))+\mathrm{C}(2,4) * \mathrm{D}\left(\mathrm{USD} \_\mathrm{RUB}(-1), 2\right)+\mathrm{C}(2,5) * \mathrm{D}(\mathrm{USD}$ JPY $(-1), 2)+\mathrm{C}(2,6)$

* D(USD_CHF $(-1), 2)+\mathrm{C}(2,7) * \mathrm{D}\left(\mathrm{EUR} \_\mathrm{USD}(-1), 2\right)+\mathrm{C}(2,8)$

$\mathrm{D}\left(\mathrm{USD} \_S A R\right)=\mathrm{A}(3,1) *\left(\mathrm{~B}(1,1) * \mathrm{BRENT}(-1)+\mathrm{B}(1,2) * \mathrm{USD} \_\mathrm{ZAR}(-1)+\mathrm{B}(1,3) *\right.$ USD_SAR$(-1)$

$+\mathrm{B}(1,4) * \mathrm{D}(\mathrm{USD}$ RUB $(-1))+\mathrm{B}(1,5) * \mathrm{D}(\mathrm{USD} J \mathrm{JPY}(-1))+\mathrm{B}(1,6) * \mathrm{D}\left(\mathrm{USD} \_\mathrm{CHF}(-1)\right)+\mathrm{B}(1,7)$

* D(EUR_USD $(-1))+\mathrm{B}(1,8))+\mathrm{C}(3,1) * \mathrm{D}(\mathrm{BRENT}(-1))+\mathrm{C}(3,2) * \mathrm{D}(\mathrm{USD} Z \mathrm{ZAR}(-1))+\mathrm{C}(3,3)$

* D(USD_SAR $(-1))+\mathrm{C}(3,4) * \mathrm{D}\left(\mathrm{USD} \_\mathrm{RUB}(-1), 2\right)+\mathrm{C}(3,5) * \mathrm{D}(\mathrm{USD}$ JPY $(-1), 2)+\mathrm{C}(3,6)$

* D(USD_CHF $(-1), 2)+\mathrm{C}(3,7) * \mathrm{D}\left(\mathrm{EUR} \_U S \mathrm{D}(-1), 2\right)+\mathrm{C}(3,8)$

$\mathrm{D}\left(\mathrm{USD} \_\mathrm{RUB}, 2\right)=\mathrm{A}(4,1) *(\mathrm{~B}(1,1) * \mathrm{BRENT}(-1)+\mathrm{B}(1,2) * \mathrm{USD} Z \mathrm{ZAR}(-1)+\mathrm{B}(1,3)$ * USD_SAR $(-1)$

$+\mathrm{B}(1,4) * \mathrm{D}\left(\mathrm{USD} \_\mathrm{RUB}(-1)\right)+\mathrm{B}(1,5) * \mathrm{D}\left(\mathrm{USD} \_\mathrm{JPY}(-1)\right)+\mathrm{B}(1,6) * \mathrm{D}\left(\mathrm{USD} \_\mathrm{CHF}(-1)\right)+\mathrm{B}(1,7)$

* D(EUR_USD $(-1))+\mathrm{B}(1,8))+\mathrm{C}(4,1) * \mathrm{D}(\mathrm{BRENT}(-1))+\mathrm{C}(4,2) * \mathrm{D}(\mathrm{USD} Z \mathrm{ZAR}(-1))+\mathrm{C}(4,3)$

* D(USD_SAR $(-1))+\mathrm{C}(4,4) * \mathrm{D}\left(\mathrm{USD} \_\mathrm{RUB}(-1), 2\right)+\mathrm{C}(4,5) * \mathrm{D}\left(\mathrm{USD} \_J P Y(-1), 2\right)+\mathrm{C}(4,6)$

* D(USD_CHF $(-1), 2)+\mathrm{C}(4,7) * \mathrm{D}($ EUR_USD $(-1), 2)+\mathrm{C}(4,8)$

$\mathrm{D}\left(\mathrm{USD} \_J P Y, 2\right)=\mathrm{A}(5,1) *(\mathrm{~B}(1,1) * \mathrm{BRENT}(-1)+\mathrm{B}(1,2)$ * USD_ZAR $(-1)+\mathrm{B}(1,3)$ * USD_SAR $(-1)$

$+\mathrm{B}(1,4) * \mathrm{D}\left(\mathrm{USD} \_\mathrm{RUB}(-1)\right)+\mathrm{B}(1,5) * \mathrm{D}\left(\mathrm{USD} \_J P Y(-1)\right)+\mathrm{B}(1,6) * \mathrm{D}\left(\mathrm{USD} \_\mathrm{CHF}(-1)\right)+\mathrm{B}(1,7)$

* D(EUR_USD $(-1))+\mathrm{B}(1,8))+\mathrm{C}(5,1) * \mathrm{D}(\mathrm{BRENT}(-1))+\mathrm{C}(5,2) * \mathrm{D}(\mathrm{USD} Z \mathrm{ZAR}(-1))+\mathrm{C}(5,3)$

* D(USD_SAR $(-1))+\mathrm{C}(5,4) * \mathrm{D}\left(\mathrm{USD} \_R U B(-1), 2\right)+\mathrm{C}(5,5) * \mathrm{D}\left(\mathrm{USD} \_J P Y(-1), 2\right)+\mathrm{C}(5,6)$

* D(USD_CHF $(-1), 2)+\mathrm{C}(5,7) * \mathrm{D}\left(\mathrm{EUR} \_\mathrm{USD}(-1), 2\right)+\mathrm{C}(5,8)$

$\mathrm{D}\left(\mathrm{USD} \_\mathrm{CHF}, 2\right)=\mathrm{A}(6,1) *(\mathrm{~B}(1,1) * \mathrm{BRENT}(-1)+\mathrm{B}(1,2) *$ USD_ZAR $(-1)+\mathrm{B}(1,3)$ * USD_SAR $(-1)$

$+\mathrm{B}(1,4)$ * $\mathrm{D}\left(\mathrm{USD} \_\mathrm{RUB}(-1)\right)+\mathrm{B}(1,5) * \mathrm{D}\left(\mathrm{USD} \_\mathrm{JPY}(-1)\right)+\mathrm{B}(1,6) * \mathrm{D}\left(\mathrm{USD} \_\mathrm{CHF}(-1)\right)+\mathrm{B}(1,7)$

* D(EUR_USD $(-1))+\mathrm{B}(1,8))+\mathrm{C}(6,1) * \mathrm{D}(\mathrm{BRENT}(-1))+\mathrm{C}(6,2) * \mathrm{D}(\mathrm{USD} Z \mathrm{ZAR}(-1))+\mathrm{C}(6,3)$

* D(USD_SAR $(-1))+\mathrm{C}(6,4) * \mathrm{D}\left(\mathrm{USD} \_\mathrm{RUB}(-1), 2\right)+\mathrm{C}(6,5) * \mathrm{D}\left(\mathrm{USD} \_J P Y(-1), 2\right)+\mathrm{C}(6,6)$

* D(USD_CHF(-1),2) + C(6,7) * D(EUR_USD $(-1), 2)+\mathrm{C}(6,8)$

$\mathrm{D}($ EUR_USD, 2$)=\mathrm{A}(7,1) *(\mathrm{~B}(1,1) * \mathrm{BRENT}(-1)+\mathrm{B}(1,2) * \mathrm{USD} Z \mathrm{ZAR}(-1)+\mathrm{B}(1,3) *$ USD_SAR $(-1)$

$+\mathrm{B}(1,4) * \mathrm{D}\left(\mathrm{USD} \_\mathrm{RUB}(-1)\right)+\mathrm{B}(1,5) * \mathrm{D}\left(\mathrm{USD} \_\mathrm{JPY}(-1)\right)+\mathrm{B}(1,6) * \mathrm{D}\left(\mathrm{USD} \_\mathrm{CHF}(-1)\right)+\mathrm{B}(1,7)$

* D(EUR_USD $(-1))+\mathrm{B}(1,8))+\mathrm{C}(7,1) * \mathrm{D}(\mathrm{BRENT}(-1))+\mathrm{C}(7,2) * \mathrm{D}(\mathrm{USD} Z \mathrm{ZAR}(-1))+\mathrm{C}(7,3)$

* D(USD_SAR $(-1))+\mathrm{C}(7,4) * \mathrm{D}\left(\mathrm{USD} \_R U B(-1), 2\right)+\mathrm{C}(7,5) * \mathrm{D}\left(\mathrm{USD} \_\mathrm{JPY}(-1), 2\right)+\mathrm{C}(7,6)$

* D(USD_CHF $(-1), 2)+\mathrm{C}(7,7) * \mathrm{D}($ EUR_USD $(-1), 2)+\mathrm{C}(7,8)$

Finally for analysing short run causality under $5 \%$ level of significance Granger test is applied. According to the results only USD/RUB Granger causes Brent with 10\% level of significance. Whereas USD/ZAR, USD/ SAR, USD/JPY, USD/CHFand EUR/USD does not Granger Cause Brent. Brent on the other hand Granger causes, USD/ZAR, USD/RUB, USD/CHF and EUR/USD. The results are given in Table 9.

\section{Discussion}

WTI and Brent oil are modelled separately to check for possible effects of exchange rates. First long term relationships are analyzed. This is done with Vector autoregression model (VAR). The stability of the model is also tested. The variables are tested for Johansen cointegration both with Trace and Max-eigenvalue methodology. There are 6 cointegrating equations for VAR model with WTI and exchange rates and 5 cointegrating equations for VAR model Brent and exchange rates. Cointegration results show that variables have long-term relationship.

Once the long term relationships are detected, short term relationships are also analyzed. The methodology for this is Granger Causality applied with a Vector Error Correction Model (VECM). For the VECM with WTI, USD/CHF, USD/SAR, USD/ZAR, and EUR/USD Granger causes WTI, WTI on the other hand Granger causes, USD/CHF, USD/RUB, USD/ZAR and EUR/USD. When applied with VECM including Brent, only USD/RUB Granger causes Brent with 10\% level of significance, whereas Brent Granger causes, USD/ZAR, USD/RUB, USD/CHF and EUR/USD. 
Table 9. Granger causality results.

\begin{tabular}{|c|c|c|c|}
\hline \multicolumn{4}{|c|}{ VEC Granger Causality/Block Exogeneity Wald Tests } \\
\hline \multicolumn{4}{|c|}{ Date: 12/21/14 Time: $16: 26$} \\
\hline \multicolumn{4}{|c|}{ Sample: 2/23/2011 9/19/2014 } \\
\hline \multicolumn{4}{|c|}{ Included observations: 897} \\
\hline \multicolumn{4}{|c|}{ Dependent variable: D(BRENT) } \\
\hline Excluded & Chi-sq & $\mathrm{df}$ & Prob. \\
\hline D(USD_ZAR) & 0.674986 & 1 & 0.4113 \\
\hline D(USD_SAR) & 0.682669 & 1 & 0.4087 \\
\hline D(USD_RUB,2) & 3.365229 & 1 & 0.0666 \\
\hline D(USD_JPY,2) & 0.954807 & 1 & 0.3285 \\
\hline D(USD_CHF,2) & 0.115260 & 1 & 0.7342 \\
\hline D(EUR_USD,2) & 0.932746 & 1 & 0.3342 \\
\hline All & 5.601936 & 6 & 0.4692 \\
\hline \multicolumn{4}{|c|}{ Dependent variable: D(USD_ZAR) } \\
\hline Excluded & Chi-sq & df & Prob. \\
\hline $\mathrm{D}(\mathrm{BRENT})$ & 42.76761 & 1 & 0.0000 \\
\hline D(USD_SAR) & 0.023423 & 1 & 0.8784 \\
\hline D(USD_RUB,2) & 0.006077 & 1 & 0.9379 \\
\hline D(USD_JPY,2) & 2.780567 & 1 & 0.0954 \\
\hline D(USD_CHF,2) & 1.843748 & 1 & 0.1745 \\
\hline D(EUR_USD,2) & 0.416966 & 1 & 0.5185 \\
\hline All & 47.78798 & 6 & 0.0000 \\
\hline \multicolumn{4}{|c|}{ Dependent variable: D(USD_SAR) } \\
\hline Excluded & Chi-sq & df & Prob. \\
\hline D(BRENT) & 2.006908 & 1 & 0.1566 \\
\hline D(USD_ZAR) & 1.510581 & 1 & 0.2191 \\
\hline D(USD_RUB,2) & 0.059348 & 1 & 0.8075 \\
\hline D(USD_JPY,2) & 0.378800 & 1 & 0.5382 \\
\hline D(USD_CHF,2) & 5.452915 & 1 & 0.0195 \\
\hline D(EUR_USD,2) & 4.504856 & 1 & 0.0338 \\
\hline All & 10.31451 & 6 & 0.1120 \\
\hline \multicolumn{4}{|c|}{ Dependent variable: D(USD_RUB,2) } \\
\hline Excluded & Chi-sq & $\mathrm{df}$ & Prob. \\
\hline D(BRENT) & 34.88765 & 1 & 0.0000 \\
\hline D(USD_ZAR) & 34.04939 & 1 & 0.0000 \\
\hline D(USD_SAR) & 0.997805 & 1 & 0.3178 \\
\hline
\end{tabular}




\section{Continued}

$\begin{array}{cc}\text { D(USD_JPY,2) } & 6.219351 \\ \text { D(USD_CHF,2) } & 25.88511 \\ \text { D(EUR_USD,2) } & 22.04458 \\ \text { All } & 105.8214\end{array}$

0.0126

0.0000

$1 \quad 0.0000$

6

0.0000

Dependent variable: D(USD_JPY,2)

$\begin{array}{cc}\text { Excluded } & \text { Chi-sq } \\ \text { D(BRENT) } & 1.452793 \\ \text { D(USD_ZAR) } & 5.494526 \\ \text { D(USD_SAR) } & 0.022154 \\ \text { D(USD_RUB,2) } & 0.164749 \\ \text { D(USD_CHF,2) } & 0.190156 \\ \text { D(EUR_USD,2) } & 1.445675 \\ & 10.37634\end{array}$

Dependent variable: D(USD_CHF,2)

Excluded

D(BRENT)

D(USD_ZAR)

D(USD_SAR)

D(USD_RUB,2)

D(USD_JPY,2)

D(EUR_USD,2)

All

\section{Excluded \\ D(BRENT)}

D(USD_ZAR)

D(USD_SAR)

D(USD_RUB,2)

D(USD_JPY,2)

D(USD_CHF,2)

All

Chi-sq
16.59352
34.12974
0.547570
0.413585
0.620430
12.04784
63.32304

Dependent variable: D(EUR_USD,2)

Chi-sq

47.46756

35.84237

3.654745

0.005313

5.757389

44.30970

144.7032$$
\text { df }
$$

Prob.

0.2281

0.0191

0.8817

0.6848

0.6628

0.2292

0.1097

6

df

Prob.

0.0000

0.0000

0.4593

0.5202

0.4309

0.0005

0.0000

6

Prob.

0.0000

0.0000

0.0559

0.9419

0.0164

0.0000

0.0000

According to the results, many currencies including EUR/USD Granger causes WTI and USD/RUB Granger causes Brent which is an expected result since Brent is not a US based oil. Both Brent and WTI Granger causes USD/CHF, USD/RUB, USD/ZAR and EUR/USD. This is indicating that although oil is traded with US Dollar, it is theoretically possible to trade oil with currencies such as Euro and Ruble.

\section{Conclusions}

In international trade most trades although not traded with USA occur with US Dollar. This leads to the phenomenon of unofficial dollarization. There are however attempts to make the trade in currencies other than US Dollar. This attempt is known as de-dollarization.

The focus of this paper is oil trade where the trade currency is US Dollar. There are attempts to make the 
trade in Euro, Ruble, Rial or Yuan. This paper focuses on the effects of oil on exchange rates of several currencies and the effects of currencies on oil. The findings indicate that there is an effect on the price of oil on currencies such as Ruble and Euro. Therefore it is theoretically possible to make the oil trade in these currencies.

Being the trade currency for oil gives USA important advantages such as increased investment in US assets. Euro is the most important rival to US Dollar for being the reserve currency when considered the wide usage and the experience of European Central Bank. This would help European Economy and would decrease the cost of funding for European Central Bank. There are however difficulties involved namely the political dominance of USA and high US reserves and production. This gives the USA opportunity to increase production and decrease the price of oil together in favor of USA with the increased use of Shale Gas which would lead to competitive advantage.

\section{References}

[1] Tas, B.K.O. and Togay, S. (2010) Optimal Monetary Policy Regime for Oil Producing Developing Economies: Implications for Post-War Iraq. Economic Modelling, 27, 1324-1325. http://dx.doi.org/10.1016/j.econmod.2010.01.001

[2] Mete, F. (2012) Liability Dollarization, Exchange Market Pressure and Fear of Floating: Empirical Evidence for Turkey. Applied Economics, 44, 1041. http://dx.doi.org/10.1080/00036846.2010.534073

[3] Berrios, R. (2006) Cost and Benefit of Ecuador's Dollarization Experience. Perspectives on Global Development and Technology, 5, 55.

[4] Togay, S. (2009) The Role of Monetary Policy in Reducing Kazakh Economy’s Dependence on Oil. Billig, 48, 239.

[5] Mentel, G. (2013) Modelling Gas Prices in Poland with an Application of Vector Autoression Method (VAR). Folia Oeconomica Stetinensia, 48-49.

[6] Permani, R. (2013) Optimal Export Tax Rates of Cocoa Beans: A Vector Error Correction Model Approach. Australian Journal of Agricultural and Resource Economics, 57, 587. http://dx.doi.org/10.1111/1467-8489.12011

[7] Lu, F., Hong, Y., Wang, S., Lai, K. and Liu, J. (2014) Time-Varying Granger Causality Tests for Applications in Global Crude Oil Markets. Energy Economics, 42, 290. http://dx.doi.org/10.1016/j.eneco.2014.01.002

[8] Zhang, Y. and Wei, Y. (2010) The Crude Oil Market and the Gold Market: Evidence for Cointegration, Causality and Price Discovery. Resources Policy, 35, 170. http://dx.doi.org/10.1016/j.resourpol.2010.05.003 